\title{
ACQUA E IRRIGAZIONE PER NUTRIRE IL PIANETA. LA REALTÀ DELLA PIANURA PADANA LOMBARDA
}

\author{
CLAUDIO GANDOLFI (*)
}

SuNTO. - Le aree pianeggianti della Lombardia sono parte di un contesto territoriale, come quello della Pianura Padana, che è il principale in Italia e tra i più importanti in Europa per quantità e per qualità delle produzioni agrarie ed alimentari. Ciò che contribuisce ulteriormente all'eccezionalità di queste aree è il retaggio legato allo sviluppo storico delle attività agricole, che trova una delle sue testimonianze più evidenti ed importanti nello straordinario sviluppo dei manufatti destinati all'irrigazione, fin da epoca medioevale. Le opere di derivazione delle acque dai principali fiumi, con l'estesissima rete di canali che da esse si originano, hanno contribuito a modellare il territorio e a disegnare il paesaggio lombardo. L'attuale assetto dei sistemi irrigui ha quindi caratteristiche uniche e molteplici aspetti positivi. D'altra parte bisogna riconoscere che la presenza di infrastrutture obsolete, il sedimentarsi di antichi diritti e l'eccesso di frammentazione degli enti di gestione rappresentano elementi negativi, particolarmente accentuati in alcune zone. Sono quindi necessari investimenti per l'innovazione delle infrastrutture e della loro gestione, ma è altrettanto indispensabile un'azione incisiva di pianificazione a livello regionale che li indirizzi con chiarezza verso obiettivi precisi, su cui si avanzano alcune proposte.

$$
* * *
$$

ABSTRACT. - The plain areas of Lombardy are part of a territorial context, such as that of the Po Valley, which is the most important in Italy and among the most important in Europe for quantity and quality of agricultural and food production. What further contributes to the exceptional nature of these areas is the historical legacy linked to the development of the agricultural activities, which finds one of its clearest and most important evidences in the extraordinary development of canals and irrigation artifacts since the Middle Ages. The diversions from the main rivers, with the extensive network of canals that they originate, have contributed to modeling the territory and shaping the Lombard landscape. The current arrangement of irrigation systems has, therefore, unique features and many positive aspects. On the other hand, one must recognize that the presence of many obsolete infrastructures, the sedimentation of ancient rights and the excessive fragmentation of management bodies are negative elements, particularly in some areas. Investments in the innovation of both the infrastructures and of their management are therefore needed, but it is equally indispensable to deploy an incisive planning action at the regional level, which addresses them towards precise objectives, on which some proposals are put forward in this paper.

(*) Dipartimento di Scienze Agrarie e Ambientali, Università degli Studi di Milano, Milano, Italia. E-mail: claudio.gandolfi@unimi.it 


\section{StATO DELl'iRRIGAZIONE IN LOMBARDiA}

\subsection{Superfici irrigate, colture e metodi irrigui}

Il territorio di bonifica ed irrigazione della Lombardia copre tutta la pianura regionale, fino alle prime pendici delle Prealpi, e comprende una superficie agricola utilizzata (SAU) di circa 700.000 ha, pari al $72 \%$ della SAU totale e il $58 \%$ della superficie territoriale regionale. La superficie irrigabile è la quasi totalità della $\mathrm{SAU}$, mentre quella irrigata è di oltre 550.000 ha, il 78\% della SAU di pianura ${ }^{1}$.

I dati Istat mostrano che la superficie irrigabile è diminuita tra il $2000^{2}$ e il 2010 per le aziende con superficie inferiore a 50 ha, mentre è aumentata per quelle con superficie maggiore ed in particolare in quelle che superano i $100 \mathrm{ha}$. Complessivamente, invece, la superficie è rimasta quasi invariata, mentre dal punto di vista della distribuzione provinciale si è avuta una leggera riduzione in quasi tutte le province ad eccezione di quelle di Brescia, Mantova e Lodi.

Tra le coltivazioni prevale il mais, con oltre la metà della SAU irrigata, seguito dal riso (circa $1 / 5$ della SAU) e quindi da prati e pascoli, altre foraggere, altri seminativi e altri cereali. La distribuzione provinciale mostra come nelle province di Brescia e Cremona la SAU irrigata sia quasi completamente occupata da mais, che prevale anche in quelle di Mantova e di Lodi; il riso, invece, domina nella provincia di Pavia (Fig. 1). Questo tipo di distribuzione ha importanti ricadute sulla richiesta idrica, sia in termini dei quantitativi complessivi che della loro distribuzione temporale.

Oltre la metà della superficie irrigata viene approvvigionata da acqua fornita da un ente irriguo con consegna turnata, mentre un altro $20 \%$ con consegna alla domanda; il resto della superficie è irrigata con acque provenienti da fonti d'approvvigionamento dirette delle aziende, prevalentemente da acque superficiali, ma in misura significativa anche acque sotterranee.

I metodi irrigui utilizzati nelle diverse zone della Pianura lombarda sono il risultato di un processo di ammodernamento ed estensione

1 Istat (2010) $6^{\circ}$ Censimento Generale dell'Agricoltura, http://censimento agricoltura.istat.it/

2 Istat (2000) $5^{\circ}$ Censimento Generale dell'Agricoltura, http://censagr.istat.it/ 
della superficie irrigata, che ha agito su un substrato storico che si è evoluto in un arco di tempo plurisecolare.

I metodi irrigui largamente prevalenti sono quelli a gravità e in particolare lo scorrimento superficiale e l'infiltrazione laterale (quasi il $60 \%$ ), a cui si aggiunge la sommersione (circa 15\%). L'aspersione è utilizzata su poco meno di un quarto circa della superficie, in genere nei territori di più recente irrigazione, a causa sia della minor disponibilità d'acqua, sia della natura e della giacitura dei terreni che rendono difficoltosa la pratica irrigua per scorrimento. Gli altri metodi ammontano al restante $2 \%$, attestando che la pratica dell'irrigazione localizzata è molto limitata, in estensione soprattutto per le colture orticole, ma con alcune esperienze importanti anche per i seminativi.

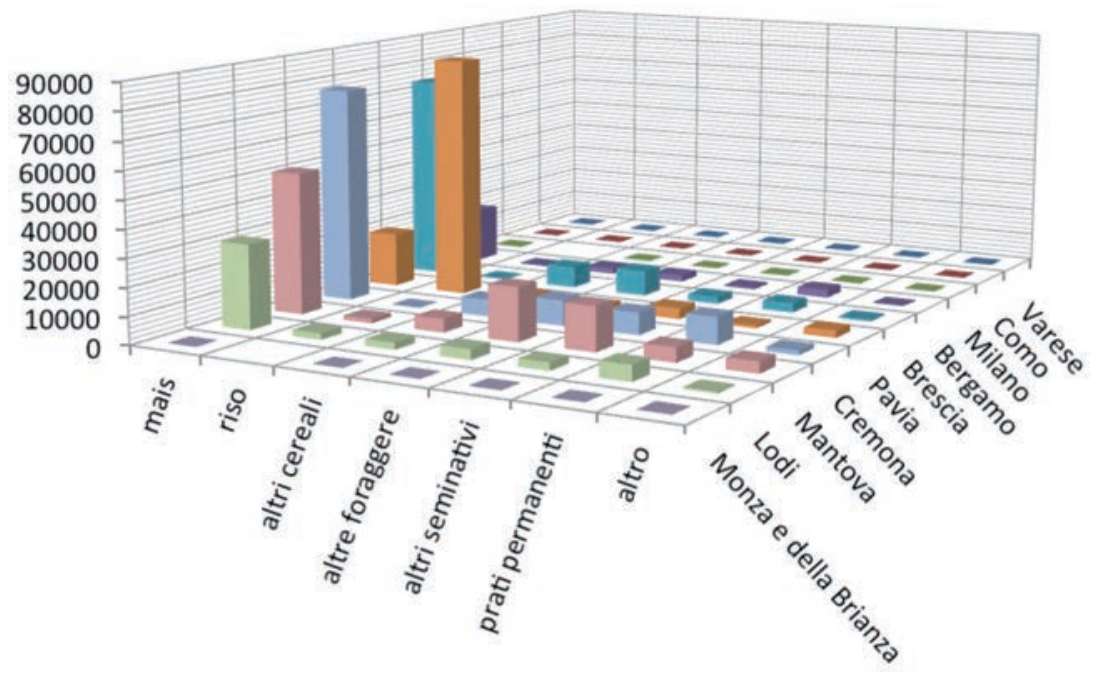

Fig. 1. Distribuzione per provincia dell'utilizzazione del suolo in pianura.

(Dati Istat, Censimento Agricoltura 2010).

Dal punto di vista della distribuzione spaziale, si evidenzia una prevalenza dello scorrimento nei territori centro-occidentali e dell'aspersione in quelli sud-orientali (Fig. 2). Caratteristiche peculiari hanno i territori sud-occidentali della pianura, tra i fiumi Sesia e Lambro, dove la presenza quasi ubiquitaria della coltura del riso si compenetra con il metodo irriguo della sommersione e con una distribuzione pressoché continua della fornitura irrigua. 


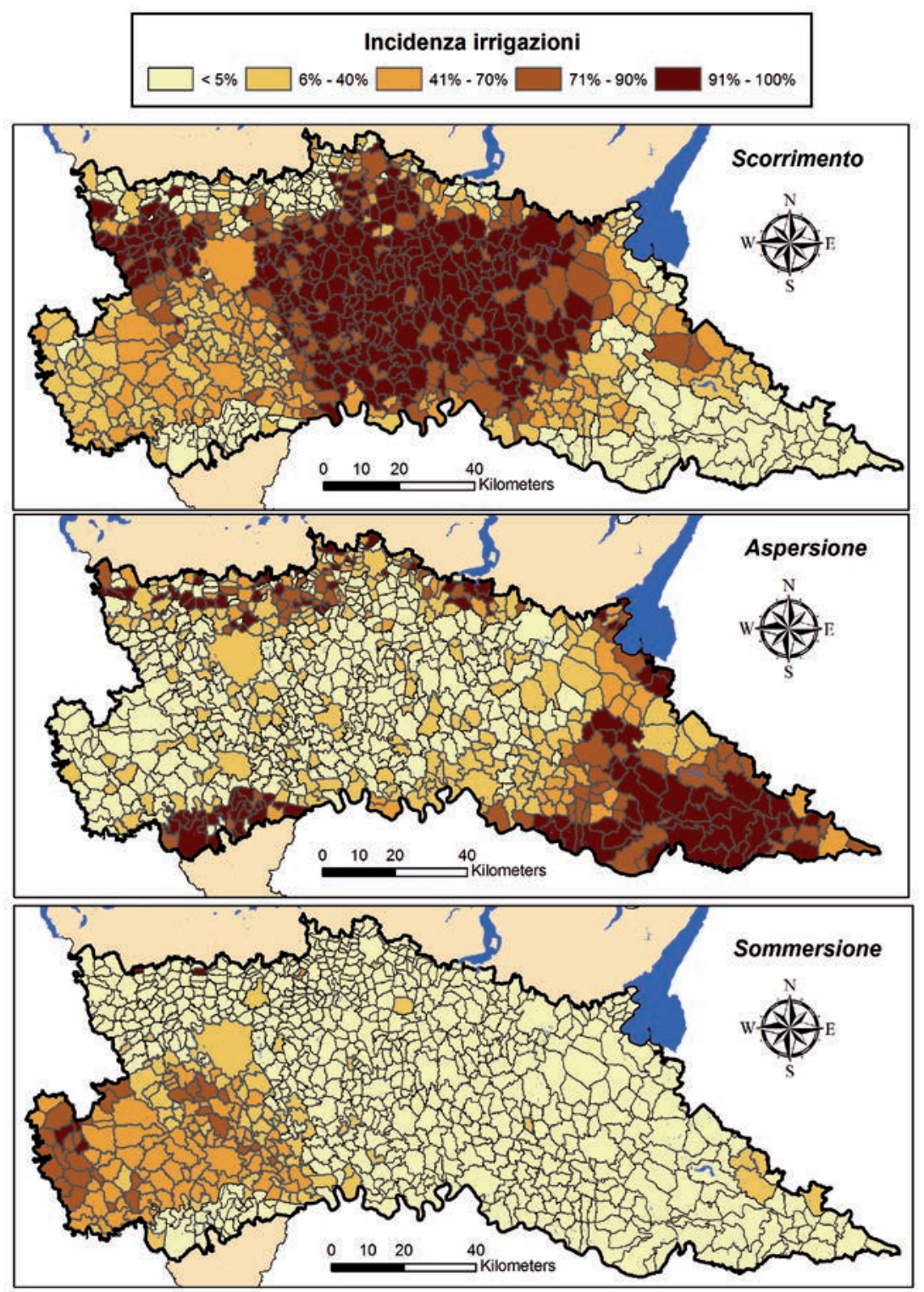

Fig. 2. Incidenza percentuale della superficie irrigata con i principali metodi. (Fonte: Istat, 2000). 
La distribuzione dei metodi rispetto alle colture (Fig. 3) evidenzia come quasi il 70\% dell'irrigazione su mais sia effettuata con il metodo dello scorrimento e poco meno del $30 \%$ con quello dell'aspersione; per il riso, invece, in quasi l' $80 \%$ della superficie si ricorre alla sommersione, e nella restante allo scorrimento (Istat, 2010).

Ragionando in termini di volumi stagionali erogati, la sommersione risulta il metodo più importante: la metà dei volumi, viene infatti erogata secondo questa modalità. Segue lo scorrimento superficiale con il 35\%, mentre l'aspersione assomma al 10\%.

In definitiva, dai dati presentati è possibile osservare che, dove è maggiore la disponibilità idrica, i terreni lo consentono e le colture non hanno particolare pregio (tipicamente mais e prato stabile), l'irrigazione per scorrimento rimane la più utilizzata. Questo è da mettere in relazione, oltre che all'origine del sistema irriguo lombardo, al fatto che tale metodo irriguo è particolarmente vantaggioso dal punto di vista degli investimenti aziendali (praticamente nulli in termini di macchine e impianti) e, in parte, anche dal punto di vista energetico, cosicché viene messo in discussione solo da particolari problemi di disponibilità e/o di organizzazione aziendale.

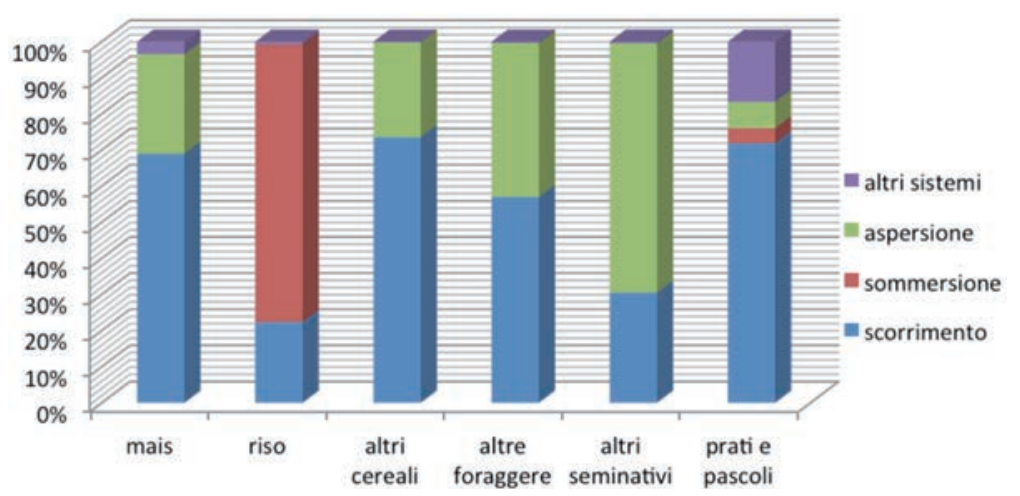

Fig. 3. Distribuzione percentuale dei metodi irrigui per le diverse colture.

Nei territori a vocazione risicola l'irrigazione per sommersione rappresenta il metodo di elezione, per la sua capacità di esercitare una fondamentale azione di termoregolazione. Grazie alla disponibilità di nuove varietà di riso, tuttavia, vi è negli ultimi anni la tendenza a ricorrere a tecniche alternative, tra cui la coltivazione in condizioni aerobi- 
che, con irrigazione turnata al pari delle altre colture irrigue. La diffusione di queste tecniche, potenzialmente vantaggiose a scala di campo, potrebbe impattare significativamente sulla circolazione idrica a scala territoriale e avere anche importanti ripercussioni sugli aspetti paesaggistici e naturalistici; le risaie della Lomellina, infatti, sono sede di un importante nodo della rete Natura 2000.

Nei territori di più recente irrigazione, come detto, prevale la tecnica dell'aspersione, vuoi per la minore disponibilità idrica, vuoi per la natura dei terreni e per la loro giacitura. In queste aree ha un seppur limitato utilizzo anche l'irrigazione localizzata, soprattutto per le colture orticole e frutticole. $\grave{E}$ anche da notare che l'irrigazione per aspersione e l'irrigazione localizzata trovano più diffuso impiego dove l'approvvigionamento è fornito dalle acque sotterranee.

\subsection{Fonti irrigue e opere di derivazione}

Le acque destinate all'irrigazione in Lombardia sono tradizionalmente derivate per gravità dai corsi d'acqua superficiali, cui si sono affiancate nell'ultimo secolo le derivazioni per sollevamento, sempre da corsi d'acqua superficiali (talvolta in concomitanza con la realizzazione degli impianti idrovori di bonifica), e gli emungimenti dalla falda freatica. Un particolare tipo di fonte è rappresentato dalle risorgive, generalmente sotto forma di fontanili, la cui alimentazione è strettamente dipendente dalle interrelazioni tra circolazione idrica superficiale e sotterranea e, in particolare, dalle perdite dai canali in terra e dai metodi d'irrigazione per scorrimento e sommersione.

La portata complessiva in concessione per derivazione da corpi idrici superficiali ammonta a circa $800 \mathrm{~m}^{3} / \mathrm{s}$ nella stagione irrigua. La gran parte della portata viene derivata mediante opere di derivazione per gravità (con concessioni per un totale di circa $700 \mathrm{~m}^{3} / \mathrm{s}$ ) e solo nei territori della bassa pianura centro-orientale, in fregio al Po, all'Oglio e al Mincio, l'approvvigionamento irriguo richiede il sollevamento delle portate prelevate dai corsi d'acqua (con concessioni per un totale di circa $100 \mathrm{~m}^{3} / \mathrm{s}$ ), seppure con modeste prevalenze. Il pompaggio è utilizzato anche in alcuni territori dell'alta pianura bergamasca e soprattutto bresciana, con prevalenze molto più elevate, ma superfici irrigate e portate complessivamente modeste (portate complessive di circa $5 \mathrm{~m}^{3} / \mathrm{s}$ ). In Tab. 1 sono riportati i valori delle portate in concessione per le principali derivazioni dai fiumi lombardi. 
Tab. 1. Sintesi dei valori di portata in concessione, portata media decadica effettivamente derivata del trimestre estivo (giugno-agosto) e portata massima delle medie decadiche.

\begin{tabular}{lc}
\hline Corso d'acqua & Portata in concessione $\left(\mathrm{m}^{3} / \mathrm{s}\right)$ \\
\hline f. Ticino & 143,5 \\
\hline f. Lambro e Olona & 15,6 \\
\hline f. Adda & 230,0 \\
\hline f. Brembo & 19,2 \\
\hline f. Serio & 21,3 \\
\hline f. Cherio & 1,2 \\
\hline f. Oglio & 86,1 \\
\hline f. Chiese & 39,3 \\
\hline f. Mella & 10,5 \\
\hline f. Mincio & 72,9 \\
\hline f. Po & 86,5 \\
\hline
\end{tabular}

Per quanto riguarda i corpi idrici sotterranei, dai dati del Catasto Utenze Idriche al 2013 risultano attive oltre 6700 concessioni per attingimento da pozzi ad uso irriguo con portate di concessione comprese tra 1 e 300 l/s. Il numero di pozzi censiti è quindi elevato e mostra un moderato aumento negli anni, ponendo problemi per un controllo diffuso delle portate prelevate. Il monitoraggio dei prelievi effettivi non è sistematico e a ciò si aggiungono fenomeni di abusivismo, di difficile quantificazione.

Le derivazioni sono concentrate innanzitutto nelle province di Brescia e Mantova (oltre il $30 \%$ in ciascuna) e secondariamente Cremona e Pavia (circa il 10\% in ciascuna); nella provincia di Bergamo l' $8 \%$ circa e il $5 \%$ in quella di Milano, mentre nelle restanti province vi sono meno dell' $1 \%$ delle concessioni. Considerando, invece, la portata media annua di concessione, su un valore complessivo di oltre $100 \mathrm{~m}^{3} / \mathrm{s}$ sull'intero territorio regionale per derivazioni da acque sotterranee ad uso irriguo, oltre la metà è nella provincia di Brescia, seguita dalla pro-

\footnotetext{
3 Solo parte lombarda.
} 
vincia di Bergamo con un quarto; Mantova e Pavia sono intorno al 5\%, Milano e Cremona tra il $3 \%$ e il $4 \%$, mentre le rimanenti province non raggiungono, sommate, l'1\%.

Una consistente fornitura è anche garantita dai ricircoli e riusi provenienti dalle risorgive e dalle colature, legate all'attività irrigua. I fontanili sono una particolare forma di risorgiva creata dall'uomo per facilitare l'affioramento delle acque delle falde più superficiali e poterle così raccogliere, indirizzare e utilizzare a scopo irriguo (Fig. 4). Si tratta quindi di un'opera dell'uomo che come tale si differenzia dalle naturali risorgive, o sorgenti di pianura. Tale pratica si può, con tutta probabilità, inserire nel quadro delle opere di bonifica successive all'anno mille e volte al drenaggio delle acque superficiali nella fascia delle risorgive per rendere i terreni coltivabili e irrigare al contempo quelli asciutti posti a valle.

I fontanili esercitano una funzione anche dal punto di vista ecologico e devono essere considerati dei veri e propri hotspot di biodiversità, dato che rappresentano uno degli ultimi habitat rifugio per molte specie vegetali e animali ecologicamente esigenti, un tempo assai diffusi nel territorio padano ed oggi in forte declino. Questa elevata diversità biologica dipende principalmente da alcune caratteristiche dell'acqua che sgorga dal sottosuolo: la temperatura, che rimane relativamente costante durante il corso dell'anno e l'oligotrofia, ossia la relativa povertà di nutrienti.

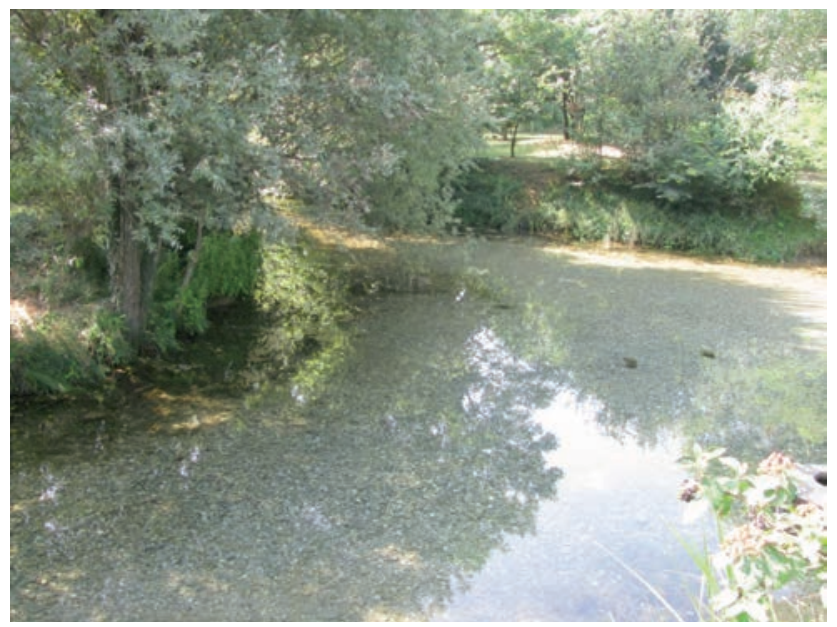

Fig. 4. Esempio di fontanile. 
La fascia delle risorgive e dei fontanili si estende nella parte settentrionale della pianura con andamento est-ovest ed ha un'ampiezza media di circa 25 chilometri. Il numero di fontanili censiti in Lombardia è di circa 1650 unità $^{4}$, distribuiti per lo più nella parte centro - occidentale della fascia; di questi 980 risultano attivi e tre quarti di essi hanno ancora un utilizzo irriguo, solo in parte soggetto a concessione (per poco più di $30 \mathrm{~m}^{3} / \mathrm{s}$ ).

\section{IL RETICOLO IDROGRAFICO LOMBARDO IN AMBITO RURALE}

La pianura lombarda è attraversata da un fittissimo reticolo idrografico artificiale che si è formato nell'ambito di una plurisecolare attività di trasformazione del territorio da parte dell'uomo, essenzialmente legata all'agricoltura ed al governo delle acque. Tale reticolo è nato con la funzione principale di fornitura irrigua o di allontanamento dell'acqua, ma ha sempre svolto anche una serie di funzioni di tipo accessorio, differenti a seconda dei contesti territoriali (la navigabilità, la sicurezza idraulica degli insediamenti, il collettamento degli scarichi civili, la formazione del paesaggio, ecc.). Funzioni che nel passato costituivano una specie di "sottoprodotto" che il mondo rurale metteva a disposizione della società intera. Con le trasformazioni attualmente in atto, sia dal punto di vista territoriale che prettamente agricolo, esse divengono sempre più numerose e diversificate e cresce l'esigenza che sia loro attribuito un valore economico. La rete idrografica rurale, inoltre, è oggi soggetta a pressioni diverse ed in un certo modo contrastanti. Da una parte vi è la necessità di garantire la massima efficienza idraulico-agraria per assicurare il risparmio idrico e ridurre i costi di gestione e manutenzione; dall'altra sorge ormai da più parti la richiesta di conservare ed aumentare la naturalità di questi canali, mediante specifiche azioni di conservazione e riqualificazione. Non ultima, la Direttiva Quadro sulle Acque definisce un nuovo contesto, dove il concetto di qualità ecologica si applica, sebbene in maniera particolare, anche ai corpi idrici artificiali quali sono i canali di irrigazione e bonifica.

4 AAVV (2012) Tutela e valorizzazione dei fontanili del territorio lombardo, Quaderni della Ricerca, n. 144, Regione Lombardia, Milano. 
L'avvio della realizzazione delle grandi infrastrutture per l'irrigazione si colloca tra la fine del XII e l'inizio del XIII secolo, nell'area dominata dalla città di Milano, con la costruzione di due grandi canali, che derivano acque dal Ticino e dall'Adda: il Naviglio Grande e il Canale Muzza. Il primo, che collega Milano al Ticino ed al Lago Maggiore, è il prototipo di numerosi altri canali italiani, che uniscono in modo originale le funzioni della navigazione e dell'irrigazione; il Muzza, che con la sua enorme portata è elemento dominante del territorio Lodigiano, era e rimane uno dei maggiori canali europei con finalità unicamente irrigue.

Questa stagione di straordinaria fioritura delle opere per l'irrigazione si apre dopo la battaglia di Legnano (1176) con la pace di Costanza (1183) tra i Comuni e l'Impero. I Comuni lombardi sono dichiarati indipendenti e liberi di scegliere la propria forma di governo, sotto la semplice protezione formale dell'Impero. Tra le condizioni stipulate a loro favore vi è la proprietà delle acque pubbliche, che si dichiarano cedute dall'Imperatore colle parole: Jura fluviorum illi cuique populo Caesares servante. L'Europa, dopo secoli di oscurità, trova così in Italia, e soprattutto in Lombardia, modelli concreti per la navigazione interna e l'irrigazione, modelli che sono poi diffusi dagli ingegneri italiani negli altri Paesi.

Nello stesso periodo anche molti altri Comuni lombardi (Cremona, Brescia, Bergamo, Treviglio) costruiscono canali navigabili e/o irrigui, più piccoli di quelli milanesi a causa delle più modeste portate dei corsi d'acqua locali, rispetto al Ticino e all'Adda, ma non inferiori per caratteristiche tecniche e capacità costruttive.

Seguiranno poi il Naviglio di Bereguardo (1457), il Naviglio della Martesana (1460), il Naviglio di Paderno (1773) e il Naviglio Pavese (1807) e tanti altri (Tab. 2). Già nel '400 quindi l'assetto dell'irrigazione in Lombardia è ampiamente consolidato e nella seconda metà del 1800 le superfici irrigate e le portate derivate nella porzione centro-meridionale della pianura lombarda si avvicinano a quelle attuali.

Si tratta di un sistema grandioso e complesso, perché multifunzionale: i canali navigabili sono anche irrigui; i canali irrigui sono anche colatori. E' una situazione unica in Europa, dove queste funzioni sono in genere separate. Fino alla metà dell'Ottocento la Lombardia aveva il primato europeo per lunghezza dei canali navigabili riferita all'unità di superficie. 
Tab. 2. Cronologia della costruzione dei canali lombardi. (Da Mainardi, 1997).

\begin{tabular}{ll}
\hline Secolo & Canale \\
\hline X & Seriola Vetra \\
\hline XI & Naviglio di Chiese, Roggia Serio \\
\hline XII & $\begin{array}{l}\text { Naviglio Grande Milanese, roggia Vettabbia, Naviglio Civico di } \\
\text { Cremona, Naviglio Vecchio di Cremona, Naviglio Vecchio Pallavicino, } \\
\text { seriola Sale (Fossa Bergamasca), roggia Morlana, roggia Serio Grande, } \\
\text { roggia Comenduna, roggia Trabattoni }\end{array}$ \\
\hline XIII & $\begin{array}{l}\text { Roggia Bogara (1217), canale Muzza (1230), roggia Brembilla (1250), } \\
\text { Seriola Vetra di Chiari (1250), seriola Vescovada (1250), naviglio }\end{array}$ \\
\hline XIV & $\begin{array}{l}\text { Roggia Moschetta-Visconti (1309), roggia Vignola, roggia Fusia (1347), } \\
\text { roggia Antegnata (1349), roggia Lonata (1365), roggia Calcinata, roggia }\end{array}$ \\
$\begin{array}{l}\text { Baiana, roggia Castellana, roggia Seriola Rudiana, roggia Terzana } \\
\text { (1389), roggione di Sartirana (1387) }\end{array}$ \\
\hline XV & $\begin{array}{l}\text { Fossa di Pozzolo (1416), roggia Montichiara (1424), roggia Rizza-Biraga } \\
\text { (1424), roggia Retorto, roggia Vailata, naviglio Martesana (1457), navi- } \\
\text { glio di Bereguardo (1457), naviglio Sforzesco (1462), naviglio Goito } \\
\text { (1462), roggia Melzi (1471), navigliaccio Binasco-Pavia (1475), roggia }\end{array}$ \\
\hline Oleggio (1493), naviglio Langosco (1493), roggia Seriate, roggia \\
Archetta, roggia Guidana, roggia Martinengo-Pradalunga
\end{tabular}

Gli antichi canali lombardi presuppongono conoscenze e capacità stupefacenti per l'epoca in cui sono stati costruiti e promuovono ulteriori sviluppi tecnico-scientifici. La grande tradizione idraulica lombarda ha prodotto, ad esempio, sistemi per la misura e la dispensa delle acque, che, nonostante siano oggi sostituiti da altri più avanzati, hanno avuto una grande importanza storicas ${ }^{5}$.

5 Di Fidio, in questo volume. 
Parallelo allo sviluppo delle infrastrutture irrigue e altrettanto importante, vi è quello del diritto delle acque e degli aspetti organizzativi. Giuseppe Bruschetti $(1823)^{6}$ scrive che "la vera storia dei canali di un dato paese si collega in molti punti colla di lui storia civile, politica ed amministrativa". E' quindi logico che in Italia, e in particolare in Lombardia, il diritto delle acque si sviluppi con grande anticipo rispetto alle altre nazioni europee. Ad esempio, nel Milanese l'istituto giuridico della servitù d'acquedotto appare già consolidato nel Quattrocento, mentre in Francia è introdotto soltanto nel 1845.

A livello organizzativo, i protagonisti della costruzione e gestione dei canali sono in parte pubblici ed in parte privati; insieme realizzano un singolare esempio d'economia mista, che per certe funzioni appare ancor oggi attuale. Lo Stato (ossia nel Medioevo i Comuni e nel Rinascimento le Signorie) costruisce e gestisce i canali principali, mentre i consorzi dei privati si occupano delle reti secondarie ed i singoli privati delle reti terziarie.

A partire dall'unità d'Italia si sviluppa un processo che vede il progressivo ridimensionamento dei consorzi volontari e l'espansione di nuovi consorzi obbligatori dei proprietari, che assumono anche le funzioni d'interesse pubblico: i Consorzi di bonifica. Il processo si compie nell'intervallo tra la legge Baccarini (R.D. n.869 del 25 giugno 1882) e la legge Serpieri (R.D. n. 215 del 13 febbraio 1933), ancora in vigore. Questo assetto non è stato modificato dall'avvio del processo di decentramento dallo Stato alle Regioni, a partire dagli anni '70 del secolo scorso, ed oggi la maggior parte degli antichi canali lombardi è formalmente attribuita al demanio regionale, ma è gestita dai Consorzi di bonifica.

La sostanziale continuità attraverso i secoli delle strutture organizzative preposte alla gestione dei canali della Lombardia ha portato al consolidarsi di modalità operative in grado di mantenere in equilibrio questo complesso sistema idraulico. Le perdite d'acqua dai canali adduttori in terra impinguano le falde ed alimentano le risorgive; in tempo di pioggia, viene ridotta la derivazione dai fiumi dei corpi d'acqua irrigui, per fare posto a quelli che sgrondano dalle campagne, contribuendo così alla difesa idraulica del territorio. I coli dai campi irrigati sono riutilizzati più a valle da altri utenti. Questa impostazione è

6 Bruschetti, G. (1823) Storia dei progetti e delle opere per l'irrigazione del Milanese, diverse edizioni. 
conforme al naturalismo della tradizione idraulica italiana - il cui emblema è il grande idraulico-medico Domenico Guglielmini (16551710) - che per alcuni aspetti precorre i moderni concetti di gestione integrata e di tutela dei corpi idrici che sono al centro della Direttiva Quadro sulle Acque 2000/60/CE e che trovano numerosi riscontri nei Programmi di Sviluppo Rurale.

E' quindi una caricatura grottesca quella che presenta l'agricoltura lombarda ignara delle tecniche sviluppate nei Paesi più avanzati e responsabile di sprechi intollerabili dell'acqua. Ovviamente è necessario subordinare nel loro insieme tutti gli usi, antichi e moderni, delle acque al rispetto degli ecosistemi fluviali ed alla salvaguardia delle acque sotterranee, ma è possibile e doveroso mettere in atto una politica di risparmi delle risorse idriche, che salvi il grande patrimonio storico-culturale ed insieme naturale e paesistico dell'irrigazione lombarda.

\section{CONSERVAZIONE DELLE RISORSE IDRICHE ED EFFICIENZA DELL'IRRIGAZIONE}

Da quanto illustrato nei paragrafi precedenti, è evidente il ruolo storico-culturale e paesaggistico-ambientale dell'agricoltura irrigua della Lombardia, in aggiunta al più ovvio valore per le produzioni. Un ruolo che si è costruito e consolidato nel tempo e che si basa su una complessa ed articolata serie di relazioni tra corsi d'acqua artificiali e naturali, tra acque superficiali e acque sotterranee, tra processi produttivi ed ecosistemi agrari.

L'efficienza della distribuzione dell'acqua attraverso il servizio irriguo, di conseguenza, non deve essere vista alla sola scala di campo, ma anche ad una scala territoriale più ampia; non deve inoltre essere valutata in termini di singolo processo produttivo, ma considerando anche le differenti funzioni e le relazioni tra i diversi comparti coinvolti.

Ovviamente questo non significa affatto che tutto debba rimanere immutato, ma che ogni valutazione dell'efficienza e ogni intervento per il suo miglioramento deve tenere conto di tale complessità. Soluzioni drastiche basate su semplificazioni eccessive, infatti, potrebbero portare a risultati di tipo opposto a quanto si vorrebbe ottenere. L'attuale assetto dei sistemi irrigui lombardi non è infatti privo di aspetti positivi. Si tratta di sistemi quasi integralmente a gravità, con fabbisogni energetici modesti, che, attraverso la loro azione secolare, hanno svolto e svol- 
gono un ruolo centrale nell'assetto idrologico del territorio. In effetti, l'irrigazione controlla la gran parte delle portate dei corsi d'acqua nei mesi estivi e, attraverso l'infiltrazione e la percolazione di parte delle acque convogliate nelle reti e distribuite sugli appezzamenti, determina in larga misura la ricarica dei corpi idrici sotterranei ed il rimpinguamento delle portate dei grandi corsi d'acqua lungo il loro percorso attraverso la pianura. Inoltre l'efficienza complessiva dell'irrigazione, a scala regionale, è significativamente superiore a quella che in generale si riscontra alla scala di azienda o di comizio irriguo, in virtù della cospicua entità dei recuperi all'interno delle stesse reti irrigue e dei ricircoli indotti dagli scambi con la falda e i corsi d'acqua appena menzionati. Infine, le caratteristiche delle reti e dei metodi irrigui utilizzati hanno contribuito a creare un paesaggio e degli ecosistemi tipici delle varie zone della pianura lombarda e costituiscono un fattore indispensabile alla sua conservazione.

D'altra parte bisogna riconoscere che la presenza di molte infrastrutture obsolete, il sedimentarsi di antichi diritti ormai anacronistici e l'eccesso di frammentazione degli enti di gestione rappresentano elementi negativi, particolarmente accentuati in alcune zone.

Indubbiamente le particolari condizioni idrologiche verificatesi in alcuni degli ultimi anni, unite ad una crescente competizione per la risorsa da parte di altri settori, a maggiori esigenze di tutela dei corpi idrici ed a variazioni significative delle politiche di sostegno e dei mercati agricoli, hanno reso più precaria la situazione dell'irrigazione consortile. Un ulteriore elemento di criticità, spesso trascurato, è costituito dall'evoluzione dell'organizzazione del lavoro nelle aziende agrarie, analoga a quella che ha avuto luogo negli altri settori produttivi, con una minore disponibilità e costi crescenti per il lavoro notturno e festivo che, tuttavia, è spesso imposto dall'organizzazione in turni ed orari della distribuzione consortile delle acque irrigue alle singole aziende. Oltre ad una presunta maggiore sicurezza della fornitura irrigua, è quindi anche la maggiore flessibilità nei tempi di accesso ad essa che ha spinto un numero crescente di imprenditori agricoli a ricercare soluzioni autonome di approvvigionamento, attraverso il prelievo diretto da falda mediante pozzi di pompaggio.

Per il governo delle risorse idriche la tendenza a sostituire l'approvvigionamento da sistemi collettivi, alimentati da corsi d'acqua superficiali, con prelievi privati dalla falda appare preoccupante per i suoi possibili effetti sulla circolazione idrica complessiva nella pianura 
e per la difficoltà nell'esercitare un'efficace azione di controllo. La dinamica molto lenta dei processi di alterazione dell'equilibrio dei corpi idrici sotterranei porta spesso ad una sottovalutazione della loro entità e ad un ritardo nella risposta, come è dimostrato, tra l'altro, dalla "emergenza falda" che ha interessato il milanese alla fine degli anni ' 90 e da numerosi casi di sovra-sfruttamento degli acquiferi verificatisi in varie parti del mondo.

L'attuale situazione della pianificazione e gestione delle risorse idriche prefigura quindi l'esigenza di un rafforzamento del ruolo dell'irrigazione collettiva, che, tuttavia, deve essere perseguito principalmente attraverso il miglioramento continuo della qualità del servizio offerto agli utenti, in termini di sicurezza dell'approvvigionamento e di flessibilità nell'accesso alla risorsa. L'urgenza di affrontare alcuni problemi impone l'adozione di misure in tempi rapidi, ma non deve far dimenticare la necessità di pianificare lo sviluppo dei sistemi irrigui su orizzonti temporali sufficientemente ampi, adeguati ad infrastrutture di questa importanza. A questo proposito vale la pena di rimarcare come l'approfondita conoscenza dei processi idrologici e delle infrastrutture idrauliche è il presupposto per l'elaborazione di un piano di misure razionale e, d'altra parte, la possibilità di realizzare interventi innovativi è strettamente legata alla diffusione ed al continuo aggiornamento delle competenze tecniche degli operatori del settore. Tra i maggiori interventi da mettere in campo vi è quindi senz'altro un vasto programma di sviluppo e diffusione delle conoscenze, che comprenda la progettazione e realizzazione di sistemi coordinati di monitoraggio e di gestione delle informazioni, l'implementazione di modelli matematici di simulazione e di sistemi di supporto alle decisioni, nonché l'istituzione di programmi di formazione permanente dei tecnici degli enti irrigui e lo sviluppo di servizi di assistenza tecnica all'irrigazione.

Vi è poi ampio spazio per la progettazione e realizzazione di azioni e di interventi mirati sia alla riduzione del fabbisogno irriguo, sia al miglioramento della regimazione delle acque. Tra i primi si possono includere la riabilitazione delle reti e la razionalizzazione delle utenze, l'automazione e il telecontrollo degli organi di manovra, la bacinizzazione dei canali e la riconversione dei metodi irrigui. Tra i secondi, il controllo dei flussi di scambio acque superficiali - acque sotterranee (anche al fine della protezione delle acque dall'inquinamento da fonti diffuse di origine agricola), la regolazione congiunta degli invasi alpini e dei grandi laghi in concomitanza di periodi siccitosi. Si tratta comunque 
quasi sempre di azioni ed interventi che presuppongo un coordinamento su unità territoriali sufficientemente ampie e significative dal punto di vista idrologico ed idraulico, con la finalità di perseguire alcuni obiettivi prioritari:

- il coordinamento nella pianificazione e gestione delle derivazioni dai corsi d'acqua principali, per raggiungere gli obiettivi imposti dalla Direttiva Quadro Europea sulle Acque 2000/60 EC;

- il coordinamento della gestione irrigua ed il riordino delle reti per: i) tutelare le acque sotterranee dall'inquinamento da fonti diffuse, in particolare nelle aree vulnerabili per i nitrati ii) mitigare gli effetti dell'accentuazione degli eventi estremi a seguito del cambiamento climatico in atto;

- lo sviluppo delle capacità progettuali e gestionali per realizzare interventi integrati sui bacini di scolo, finalizzati alla riduzione della produzione di deflussi superficiali, al controllo del rischio idraulico e al miglioramento della qualità ecologica dei corpi idrici;

- lo sviluppo delle capacità progettuali e gestionali ai fini della tutela e della valorizzazione ambientale, attraverso azioni diversificate quali la riqualificazione dei tratti rilevanti delle reti irrigue e la rivitalizzazione del sistema dei fontanili -, da inquadrarsi nel contesto della rete ecologica regionale e per conseguire gli obiettivi della già citata Direttiva in relazione ai corpi idrici artificiali. 